\title{
Polarization-Insensitive Hybrid Plasmonic Waveguide Design for Evanescent Field Absorption Gas Sensor
}

\author{
Nikolay Lvovich KAZANSKIY, ${ }^{1,2}$, Svetlana Nikolaevna KHONINA ${ }^{1,2}$, and \\ Muhammad Ali BUTT ${ }^{1,3^{*}}$
}

\author{
${ }^{1}$ Department of Technical Cybernetics, Samara National Research University, Moskovskoye Shosse 34, Samara 443086, \\ Russia \\ ${ }^{2}$ Institute of RAS-Branch of the FSRC "Crystallography and Photonics" RAS, Molodogvardeiskaya 151, Samara 443001, \\ Russia \\ ${ }^{3}$ Institute of Microelectronics and Optoelectronics, Warsaw University of Technology, Koszykowa 75, Warszawa 00-662, \\ Poland \\ *Corresponding author: Muhammad Ali BUTT_E-mail: ali_ciit_engineer@yahoo.com
}

\begin{abstract}
We propose a polarization-insensitive design of a hybrid plasmonic waveguide (HPWG) optimized at the $3.392 \mu \mathrm{m}$ wavelength which corresponds to the absorption line of methane gas. The waveguide design is capable of providing high mode sensitivity $\left(S_{\text {mode }}\right)$ and evanescent field ratio $(E F R)$ for both transverse electric (TE) and transverse magnetic (TM) hybrid modes. The modal analysis of the waveguide is performed via 2-dimension (2D) and 3-dimension (3D) finite element methods (FEMs). At optimized waveguide parameters, $S_{\text {mode }}$ and $E F R$ of 0.94 and 0.704 , can be obtained for the TE hybrid mode, respectively, whereas the TM hybrid mode can offer $S_{\text {mode }}$ and $E F R$ of 0.86 and 0.67 , respectively. The TE and TM hybrid modes power dissipation of $\sim 3 \mathrm{~dB}$ can be obtained for a $20-\mu \mathrm{m}$-long hybrid plasmonic waveguide at the $60 \%$ gas concentration. We believe that the highly sensitive waveguide scheme proposed in this work overcomes the limitation of the polarization controlled light and can be utilized in gas sensing applications.
\end{abstract}

Keywords: Hybrid plasmonic waveguide; finite element method; methane gas; evanescent field absorption gas sensor; polarization-insensitive

Citation: Nikolay Lvovich KAZANSKIY, Svetlana Nikolaevna KHONINA, and Muhammad Ali BUTT, "Polarization-Insensitive Hybrid Plasmonic Waveguide Design for Evanescent Field Absorption Gas Sensor," Photonic Sensors, 2021, 11(3): 279-290.

\section{Introduction}

Optical sensors have several applications in various fields, such as medicine, chemistry, biology, health, and safety requiring compact, reliable, and yet cost-effective optical devices [1-4]. Due to the high accuracy, the sensitivity of recognition, and detection of chemical products, optical gas sensors have been extensively investigated in the field of chemistry [5-7]. Poisonous and dangerous gases in an atmosphere exposing human health to danger and even leading to the casualties are increasingly revealing the need for the highly sensitive optical sensors to detect toxic gases [8]. Methane $\left(\mathrm{CH}_{4}\right)$ is an extremely combustible gas that has the potential for being mixed with other chemicals at very low

Received: 10 March 2020 / Revised: 24 July 2020

(C) The Author(s) 2020. This article is published with open access at Springerlink.com

DOI: $10.1007 / \mathrm{s} 13320-020-0601-6$

Article type: Regular 
levels of about $5 \%$ and is at the risk of explosion [9]. Normally, it has a foul odour when being mixed with hydrogen sulfide $\left(\mathrm{H}_{2} \mathrm{~S}\right)$ that could constitute a hazardous alarm for an agonizing explosion. On the other hand, methane gas itself is not detrimental but it has a high toxicity potential when being mixed with certain substances. Alternatively, $\mathrm{CH}_{4}$ gas is one of the most useful fossil fuels extracted naturally from the ground [10], making it possible to classify optical gas sensors as an explorer capable of detecting $\mathrm{CH}_{4}$ gas resources.

There are several waveguide (WG) schemes based on different platforms that can be utilized to realize the gas sensors [11-13]. Lately, silicon on insulator (SOI) emerged as an extremely attractive platform for integrated optics $[14,15]$. The devices based on the SOI platform are highly polarization-dependent due to high contract between silicon $(n=3.45$ at $1550 \mathrm{~nm})$ and silica $(n=1.45$ at $1550 \mathrm{~nm}$ ). The polarization of light injected in a standard optical fiber can change arbitrarily which can influence the proper functioning of the integrated chip if the fiber is directly coupled to the SOI devices [16]. One possible way to solve this problem is to split the incoming light into two separate polarizations and process them independently, which may raise the complexity and size of the optical system. Another approach is to develop polarization-insensitive SOI WGs [17-19] which will be realistic for on-chip optical interconnect applications, where there is no straightforward way to manage the polarization state of light.

For highly responsive evanescent field absorption gas sensors, the overlap of the evanescent field with the surrounding medium should be high. The evanescent field of the propagating mode can be enhanced by reducing the standard SOI WG dimensions. However, it will lead to the high propagation loss of the WG. Moreover, the ability of these WGs to confine light is limited by diffraction. Plasmonic WGs propagate the surface plasmons wave at the dielectric-metal interface which is evanescently confined in the direction perpendicular to the propagation [20-22]. These WGs can confine the light to the subwavelength region as their light confining ability is not affected by the diffraction limit [23]. However, these WGs put up with high propagation loss due to the presence of the metal as the main building block of the WG [24]. There are several other applications based on the principle of plasmonics [25-27]. The hybrid plasmonic WG (HPWG) structure can provide a way out related to the low evanescent field and high propagation loss. This WG is composed of a thin layer of the low refractive index material (such as $\mathrm{SiO}_{2}$ ) sandwiched between the high refractive index material (for example $\mathrm{Si}$ ) and metal (typically $\mathrm{Ag}$ or $\mathrm{Au}$ ). The HPWG can be designed in horizontal or vertical configurations capable of supporting (only one polarization at a time) the transverse electric (TE) and transverse magnetic (TM) hybrid modes, respectively. The marriage between the horizontal and the vertical WG configurations allows the possibility of utilizing these WGs free of the polarization state. In our previous works [11-13], we studied an evanescent field ratio $(E F R)$ of the polarization-dependent strip, rib, and slot WG structures, which offered EFRs of 0.55, 0.5, and 0.47, respectively. Here, we propose a novel design of the polarization-insensitive HPWG which shows a significantly higher EFR than one obtained in the conventional SOI WGs.

\section{WG scheme}

The proposed WG scheme is composed of a horizontal HPWG structure formed by a subwavelength low refractive index material layer sandwiched between a high index material and a metal layer. This arrangement of layers provides the confinement of the TM hybrid mode. Silicon dioxide, silicon, and gold are selected as the low index material, high index material, and metal, respectively. The refractive indices of silicon and 
silicon dioxide at $3.392 \mu \mathrm{m}$ used in this work are 3.4294 [28] and 1.4090 [29], respectively. For gold, the refractive indices along with the extinction coefficient at $3.392 \mu \mathrm{m}$ are 1.4132 and 23.382 , respectively [30]. The layer thicknesses of the silicon core, silicon dioxide, and the top gold are denoted as $H_{\text {core }}, H_{\mathrm{SiO}_{2}}$, and $H_{\text {top_Au }}$, respectively. The width of the WG core is represented as $W_{\text {core }}$. Besides, a thin $\mathrm{Au}$ layer of height $\left(H_{\text {rail-Au }}\right)$ is deposited on both sides of the WG separated by a nano-gap $(\mathrm{g})$ to provide optical confinement of the TE hybrid mode. The modal analysis of the WG is simplified by fixing two geometric parameters: (1) $H_{\text {rail-Au }}=H_{\text {core }}$ and (2) $W_{\text {core }}=H_{\text {core }}$. In the rest of the paper, $W_{\text {core }}=H_{\text {core }}$ is represented as variable $X$ which has an equal control on both the TE and TM hybrid modes. The schematic representation of the HPWG is shown in Fig. 1. Inset shows the E-field distribution of the TE and TM hybrid modes at $3.392 \mu \mathrm{m}$ which is taken in the $x y$-plane (at $H_{\mathrm{SiO}_{2}} / 2$ ) and $z y$-plane (at $W_{\text {core }} / 2$ ), respectively. The geometric parameters of the WG used in this representation are as follows: $X=H_{\text {rail-Au }}=200 \mathrm{~nm}, H_{\text {top-Au }}=g=$ $H_{\mathrm{SiO}_{2}}=50 \mathrm{~nm}$.

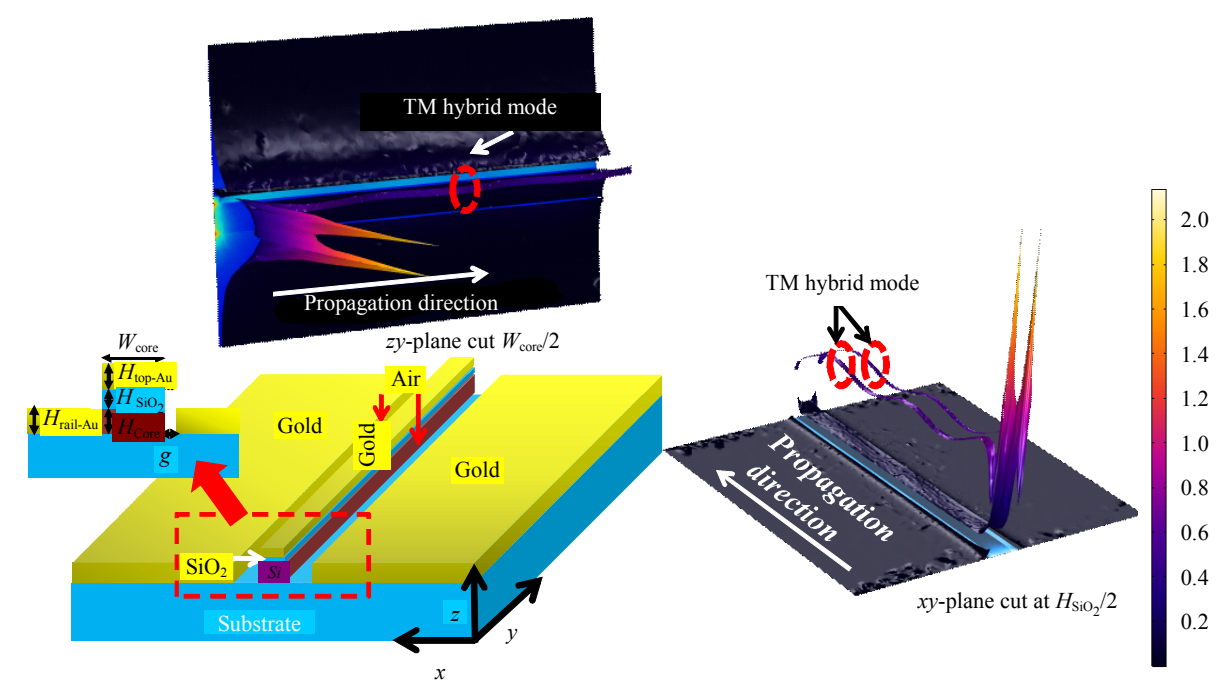

Fig. 1 Graphical representation of the polarization-insensitive HPWG design. Inset shows the 3-dimension (3D) E-field distribution of TE (bottom-right) and TM (top-left) polarized lights in the WG geometry.

The effective refractive index $\left(n_{\text {eff }}\right)$ and mode sensitivity $\left(S_{\text {mode }}\right)$ study of the HPWG design are carried out via a 2-dimension (2D) finite element method (FEM)-based model in COMSOL Multiphysics 5.1. The electromagnetic wave (EM-wave) frequency domain is elected as the physics interface and the modal analysis is added to the study. Whereas, EFR is investigated with the help of the 3D model in the aforementioned module. In COMSOL simulations, the WG geometry is divided into triangular mesh elements with a "very fine" mesh grid size for the whole design. The computational power of the system is one of the limiting factors which ensure how swiftly simulations can be carried out. We have used the system with i5 core central processing unit (CPU) at $2.5 \mathrm{GHz}$ and $64 \mathrm{~GB}$ random access memories (RAMs). For wave propagation systems, it is always desired to model a domain with open boundaries of the computational domain as it allows the EM wave to pass without any reflection. The open geometry is estimated by assigning a scattering boundary conditions (SBC) at the outer edges of the simulation window.

The E-field distribution of the TE and TM hybrid modes is visualized in a $10-\mu \mathrm{m}$-long HPWG, as shown in Fig. 2. The propagation plane is cut with an interval of $1.5 \mu \mathrm{m}$ to expose the E-field all the 
way through the WG. It can be seen that the TE hybrid mode is confined in the air gaps on both sides of the $\mathrm{Si}$ core whereas the TM hybrid mode is located in the low index material $\left(\mathrm{SiO}_{2}\right)$ sub-wavelength layer sandwiched between the $\mathrm{Si}$ core and Au layer on the top. The geometric parameters of the WG are the same as used in Fig. 1.

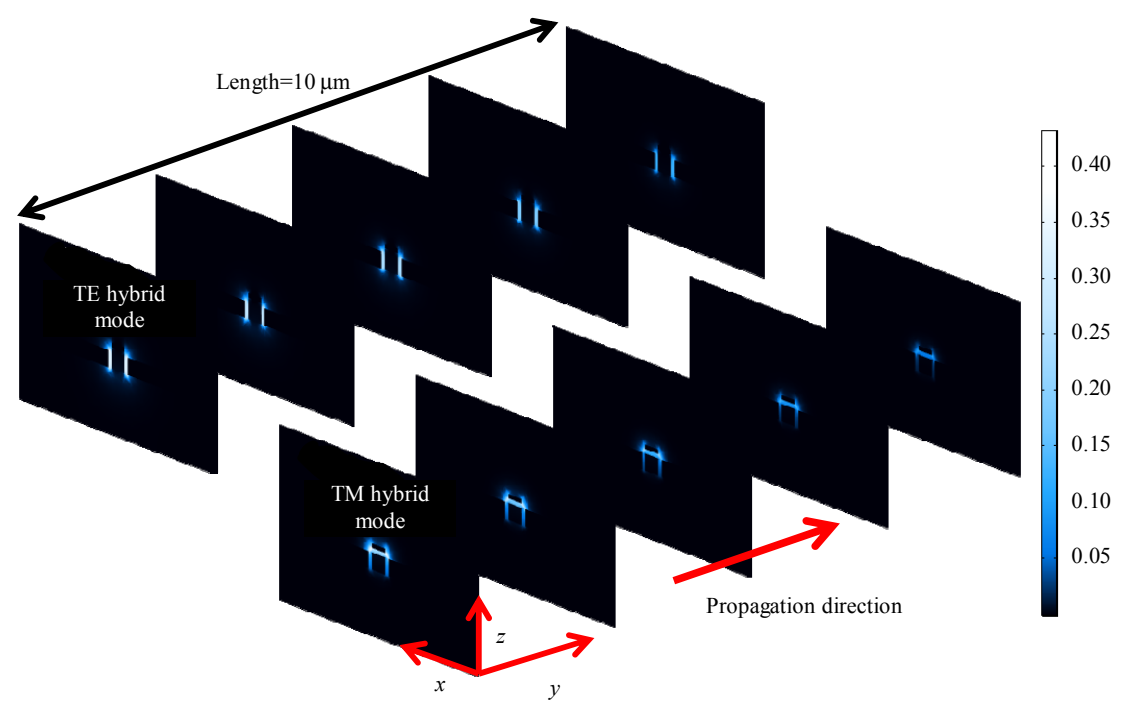

Fig. 2 E-field distribution of the propagating mode in a $10-\mu \mathrm{m}-$ long WG structure where $X=200 \mathrm{~nm}$ : (a) TE hybrid mode and (b) TM hybrid mode. The plane is cut along the $x z$-plane with an interval of $1.5 \mu \mathrm{m}$.

\section{Effective refractive index estimation of the HPWG}

The sensing mechanism in optical WGs is based on the contact between the electric field of the guided mode and the targeted sensing material. There are two vital phenomena such as EFR and $S_{\text {mode }}$ of the propagating mode to be considered while designing the sensitive device. As a general rule, the light-matter interaction is determined by the amount of the evanescent electrical field confinement around the WG core. For that reason, $E F R$ should be calculated to understand the fraction of light interacting with the ambient medium. EFR is the ratio of the power in the cover medium to the total power of the guided mode. Homogenous sensing is the best sensing method to detect variations in the concentration of gas analytes or chemical solutions present in the ambient medium. This mechanism determines the mode sensitivity of the optical WG by changing the refractive index of the analyte (ambient medium) resulting in the change of the effective refractive index ( $\left.n_{\text {eff }}\right)$ of the propagating mode. The real and imaginary parts of $n_{\text {eff }}$ of the TE and TM hybrid modes are calculated for $X$ in the range of $200 \mathrm{~nm}-1000 \mathrm{~nm}$. Additionally, the influence of other parameters such as $g, H_{\mathrm{SiO}_{2}}$, and $H_{\mathrm{Au}}$ are also taken into consideration, which seems to have less control on $n_{\text {eff. }}$ The real part of $n_{\text {eff }}$ for both the hybrid modes increases with an increase in the Si core size, i.e., $X$ due to the gradual formation of the dielectric mode as shown in Fig. 3. The imaginary part of $n_{\text {eff }}\left[\operatorname{Im}\left(n_{\text {eff }}\right)\right]$ also shows a strong dependence on the WG geometry which reduces significantly as $X$ increases. The reduction in $\operatorname{Im}\left(n_{\text {eff }}\right)$ signifies that the system has low loss which can be useful in the realization of the WGs with a longer propagation length. The loss associated with the TE hybrid mode is significantly smaller than that with the TM hybrid mode as shown in Fig. 3. This is because the TM hybrid mode is confined in a low-index $\mathrm{SiO}_{2}(n=1.44)$ layer which has some absorption in the mid-infrared band whereas the TE hybrid mode is concentrated in two 
air slots $(n=1.0)$.

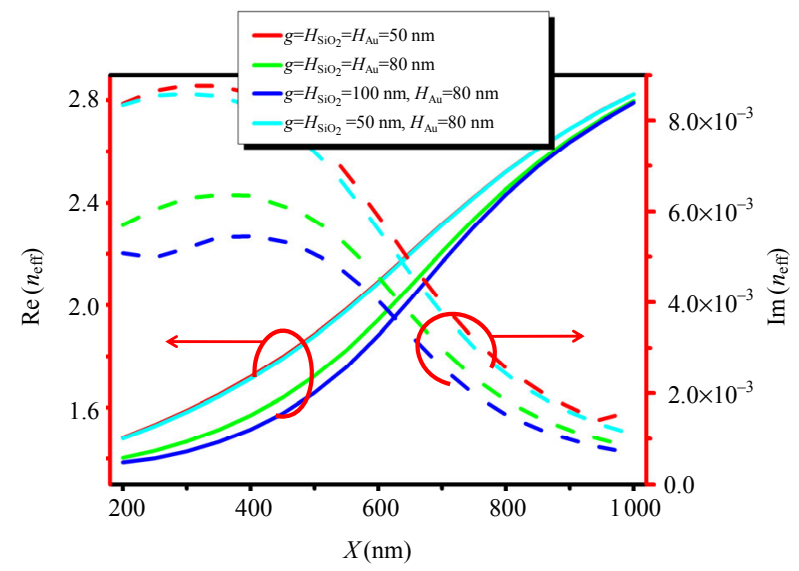

(a)

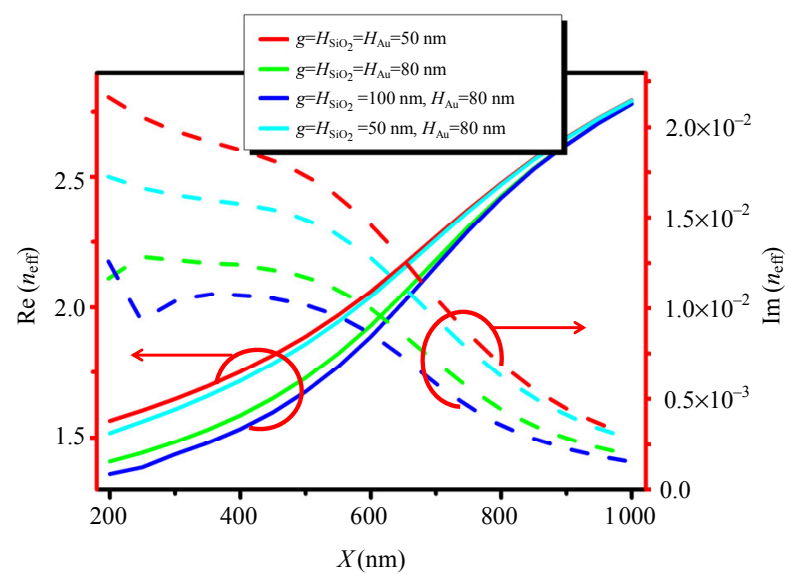

(b)

Fig. 3 Real and imaginary parts of the effective refractive index of HPWG: (a) TE hybrid mode and (b) TM hybrid mode.

\section{Estimation of propagation loss and electric field intensity}

The modal propagation loss of the HPWG is directly linked to $\operatorname{Im}\left(n_{\text {eff }}\right)$ which is calculated by using the following expression:

$$
\operatorname{Loss}\left(\frac{\mathrm{dB}}{\mu \mathrm{m}}\right)=2 K_{o} \times \operatorname{Im}\left(n_{\text {eff }}\right) \times 4.34
$$

where $K_{o}$ is $\frac{2 \pi}{\lambda_{o}}$, and $\lambda_{o}$ is the vacuum wavelength $(\mu \mathrm{m})$. It is quite evident from the equation that a smaller $n_{\text {eff }}$ will provide a lower propagation loss. From Fig. 4(a), it can be seen that the TM hybrid mode has a higher propagation loss as compared with the TE hybrid mode. This is because the TE hybrid mode propagates in nano-slots (air) whereas the TM hybrid mode is formed in a thin layer of the $\mathrm{SiO}_{2}$ layer. The $\operatorname{Im}\left(n_{\text {eff }}\right)$ of both the modes decreases as $X$ increases which eventually reduces the propagation loss. Other geometric parameters such as $g, H_{\mathrm{SiO}_{2}}$, and $H_{\mathrm{Au}}$ also influence the propagation loss of the WG which is quite visible in Fig. 4. The propagation losses of TE and TM hybrid modes are obtained in the range of $0.0107 \mathrm{~dB} / \mu \mathrm{m}$ $0.1340 \mathrm{~dB} / \mu \mathrm{m}$ and $0.027 \mathrm{~dB} / \mu \mathrm{m}-0.347 \mathrm{~dB} / \mu \mathrm{m}$, respectively. These losses can be further reduced by increasing $X$ at the cost of low EFR. However, we aim to keep $E F R$ as high as possible and the propagation loss can be compensated by using a high input power light source or small length of the WG.

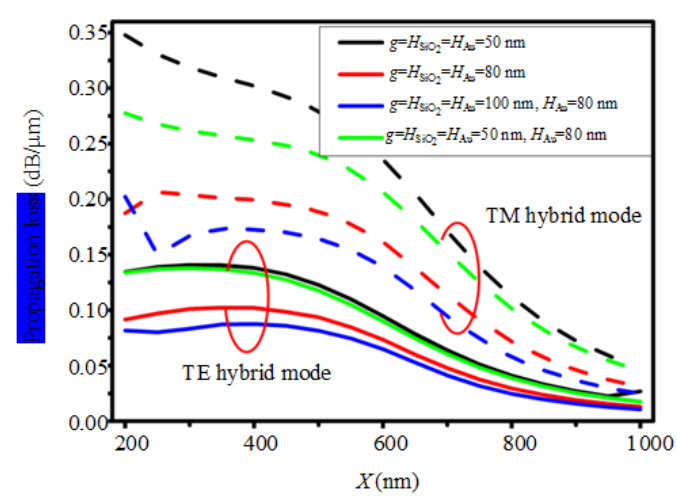

(a)

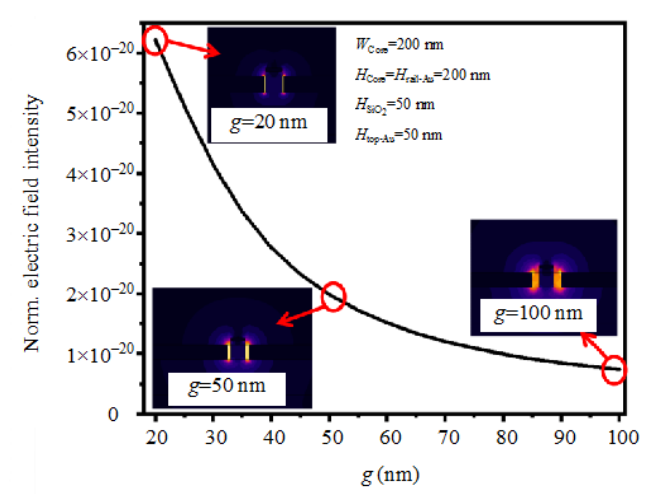

(b)

Fig. 4 Calculation of (a) modal propagation loss vs WG geometry and (b) dependence of the electric field intensity on nano-slot $(g)$.

Moreover, the electric field intensity of the TE hybrid mode in nano-slots is also investigated by 
varying $g$ in the range of $20 \mathrm{~nm}-100 \mathrm{~nm}$. Strong modal intensity can be obtained at narrow $g$, i.e., $20 \mathrm{~nm}$ and decays exponentially as $g$ approaches to $100 \mathrm{~nm}$ as shown in Fig. 4(b). However, tight modal confinement leads to a higher modal loss of the WG. Therefore, $g$ should be selected where both modal intensity and propagation losses are optimized.

\section{Mode sensitivity analysis of TE and TM hybrid mode}

$S_{\text {mode }}$ is an essential parameter which should be taken into consideration while designing sensing devices [31]. $S_{\text {mode }}$ is evaluated for the geometric parameters of the WG and is calculated by using the following expression:

$$
S_{\text {mode }}=\frac{n_{\text {eff } 2}-n_{\text {eff } 1}}{n_{2}-n_{1}}
$$

where $n_{\text {eff2 }}$ is the effective refractive index at the refractive index of the analyte under consideration $\left(n_{2}\right)$, and $n_{\text {effl }}$ is the effective refractive index at the refractive index of the medium $\left(n_{1}\right)$. In this analysis, $n_{1}$ is fixed at 1.3 whereas $n_{2}$ is varied between 1.34 and 1.4 to calculate the sensitivity of the TE and TM hybrid modes as shown in Figs. 4 and 5, respectively. $S_{\text {mode }}$ is almost stable for the range of ambient refractive indices but progressively increases as $X$ decreases as shown in Fig. 5(a). The maximum $S_{\text {mode }}=0.94$ of the TE hybrid mode is obtained at $X=$ $400 \mathrm{~nm}$ which rapidly decreases to 0.24 as $X$ approaches to $1000 \mathrm{~nm}$. The E-field distributions of the hybrid mode for $X=500 \mathrm{~nm}$ and $X=700 \mathrm{~nm}$ are shown in Figs. 5(b) and 5(c). At $X \leq 500 \mathrm{~nm}$, the WG is capable of supporting only the hybrid mode confined in the low refractive index material/nano-slot. However, at $X \geq 600 \mathrm{~nm}$, the WG geometry is large enough to facilitate the formation of the dielectric mode in the $\mathrm{Si}$ core. The mode power of the hybrid mode is partially transferred to the dielectric mode. This is the point when $S_{\text {mode }}$ and EFR of the hybrid mode decrease rapidly. The line graph of the E-field distribution of the TE hybrid mode is taken along the horizontal axis in the middle of the WG $\left(H_{\text {core }} / 2\right)$ as shown in the insets of Figs. 5(b) and 5(c). At $X=500 \mathrm{~nm}$, the TE hybrid mode has an equal mode power distribution in the nano-slots and almost zero power in the silicon core. Whereas, the WG geometry is big enough at $X=700 \mathrm{~nm}$ to facilitate the formation of the dielectric mode in the Si core which raises the power level in the middle of the line graph.

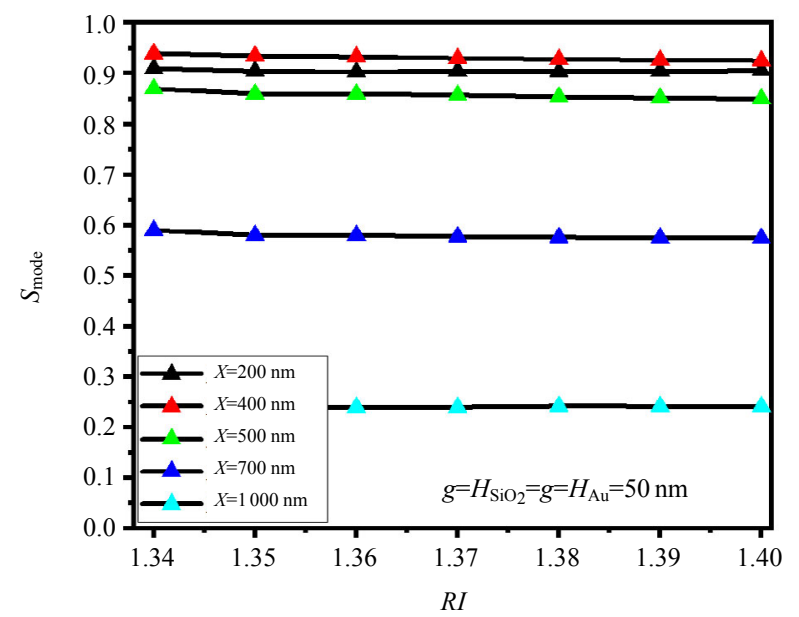

(a)
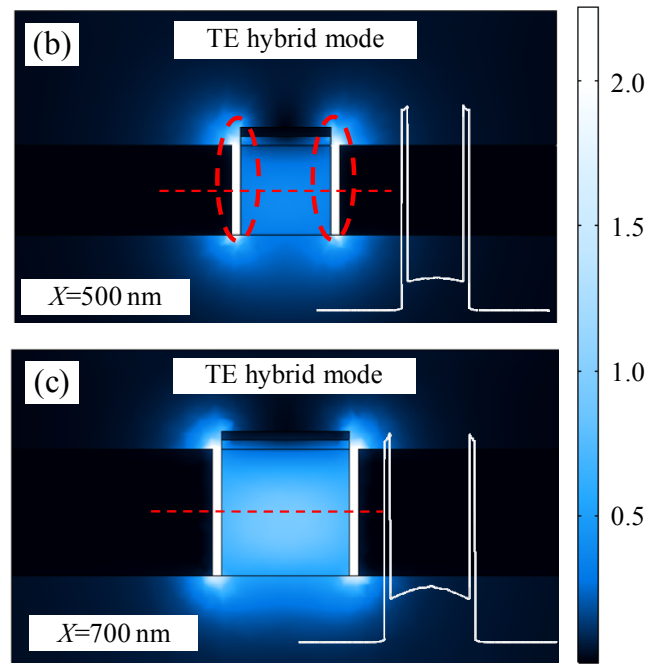

Fig. 5 Calculation of (a) mode sensitivity of the TE hybrid mode, (b) E-field distribution in the HPWG at $X=500 \mathrm{~nm}$, and (c) E-field distribution in the HPWG at $X=700 \mathrm{~nm}$. The remaining WG parameters such as $H_{\mathrm{Au}}, g$, and $H_{\mathrm{SiO}_{2}}$ are fixed at $50 \mathrm{~nm}$. Insets show the line graphs of the TE hybrid mode.

The TM hybrid mode is also highly influenced by $X$ and falls abruptly as there is an increase in $X$ as shown in Fig. 6(a). Therefore, WG geometry should be optimized where both the TE and TM hybrid 
modes possess high mode sensitivity. For instance, $S_{\text {mode }}$ as high as 0.91 and 0.86 can be obtained for the TE and TM hybrid modes, respectively for the WG geometric parameters of $X=200 \mathrm{~nm}, g=H_{\mathrm{SiO}_{2}}=$ $H_{\mathrm{Au}}=50 \mathrm{~nm}$. That is why we can use these geometric parameters for the designing of a WG for gas sensing applications. The line graph of the E-field distribution of the TM hybrid mode is taken

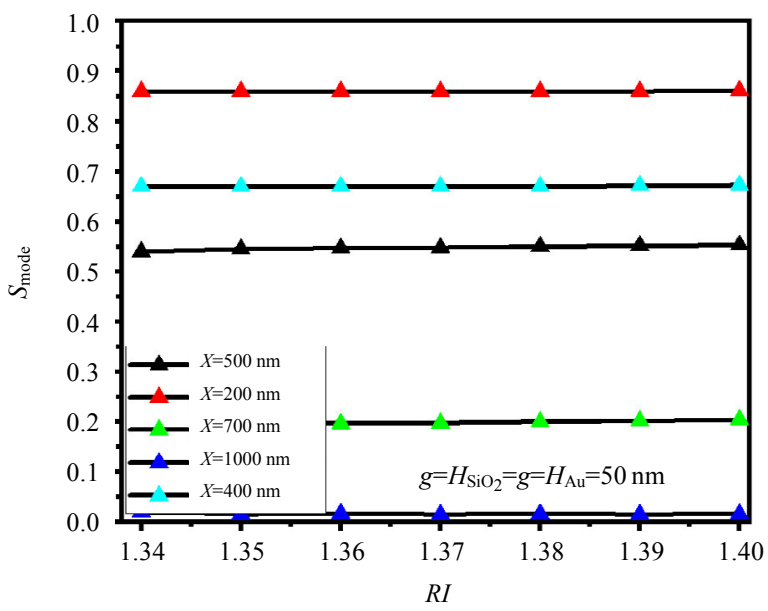

(a) along the vertical axis in the middle of the WG $\left(W_{\text {core }} / 2\right)$ as shown in the insets of Figs. 6(b) and 6(c). The TM hybrid mode has an asymmetric field distribution as the E-field is only confined in the low index medium sandwiched between the silicon layer and gold layer on the top. At $X=700 \mathrm{~nm}$, the mode power in the silicon core increases which can be visualized in the line graph.

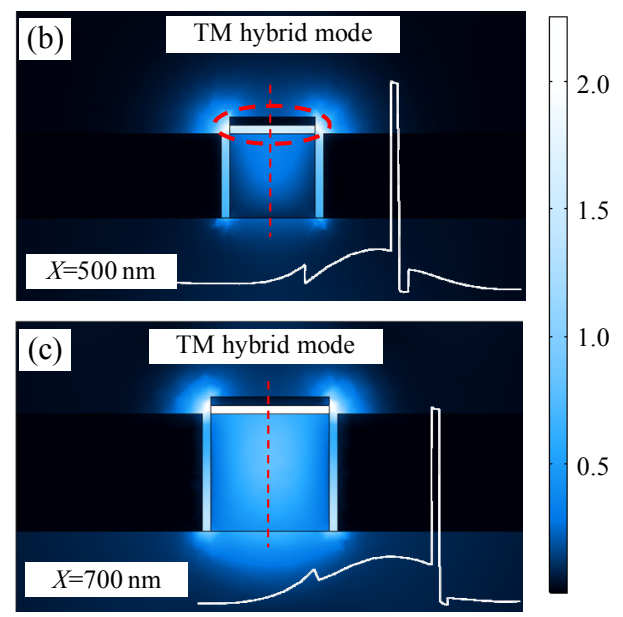

Fig. 6 Calculation of (a) mode sensitivity of the TM hybrid mode, (b) E-field distribution in the HPWG at $X=500 \mathrm{~nm}$, and (c) E-field distribution in the HPWG at $X=700 \mathrm{~nm}$. The remaining WG parameters such as $H_{\mathrm{Au}}, g$, and $H_{\mathrm{SiO}_{2}}$ are fixed at $50 \mathrm{~nm}$. Insets show the line graphs of the hybrid modes.

\section{Evanescent field ratio analysis of the HPWG}

Every gas has a characteristic absorption peak which is considered as a benchmark for that particular gas. When the WG sensor is exposed to a gaseous medium, the evanescent field interacting with that gas results in a power decay of the transmitting mode if it correlates with the absorption line of the measurand gas. The decay in power can be expressed according to Lambert-Beer's law [32-34], which normally depends on the gas concentration, length, and EFR of the WG. That is why $E F R$ is a vital parameter for the apprehension of gas sensors based on the evanescent field absorption $[35,36]$. EFR is the ratio of the intensity integration of the desired region (upper cladding + nano-slot) and overall intensity integration of the
WG structure which is expressed as

$$
E F R=\frac{\iiint_{\text {desired }}|E(x, y, z)|^{2} \mathrm{~d} x \mathrm{~d} y \mathrm{~d} z}{\iiint_{\text {total }}|E(x, y, z)|^{2} \mathrm{~d} x \mathrm{~d} y \mathrm{~d} z} .
$$

$E F R$ of the TE and TM hybrid modes largely depends on the WG geometry. WG structures with large geometric parameters have the strong mode confinement and possess the low evanescent field in the surrounding of the core. Therefore, it is necessary to calculate $E F R$ of the WG before utilizing it for sensing applications. For the TE hybrid mode, EFR has a strong dependence on $g$ ( $H_{\mathrm{Au}}$ and $H_{\mathrm{SiO}_{2}}$ do not matter too much) which increases as $g$ decreases from $100 \mathrm{~nm}$ to $50 \mathrm{~nm}$ as shown in Fig. 7(a). This is because the field confinement is stronger in smaller $g$. At $X>600 \mathrm{~nm}$, the dielectric mode starts to appear in the silicon core which kills the mode power of the hybrid mode, 
consequently, EFR falls abruptly. Similarly, the TM hybrid mode is highly influenced by $X$ and least affected by other parameters such as $H_{\mathrm{SiO}_{2}}, g$, and $H_{\mathrm{Au}}$ as shown in Fig. 7(b). The highest EFRs of the TE and TM hybrid modes are obtained at 0.75 and 0.61 , respectively for the WG length of $3 \mu \mathrm{m}$.

The dependence of EFR on the length of the WG is also determined. The geometric parameters of the WG, such as $X=200 \mathrm{~nm}$ and $H_{\mathrm{SiO}_{2}}=g=H_{\mathrm{Au}}=$

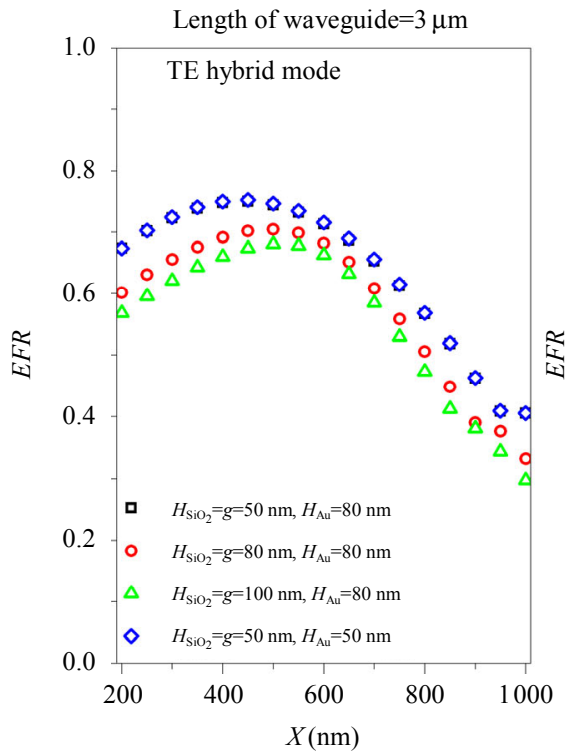

(a)
$50 \mathrm{~nm}$, are used to calculate $E F R$ of both the hybrid modes. The length of the WG is varied between $3 \mu \mathrm{m}$ and $20 \mu \mathrm{m}$. EFR of the hybrid modes increases (0.67 - 0.7 for the TE hybrid mode and $0.61-0.67$ for the TM hybrid mode) with an increase in the length $(3 \mu \mathrm{m}$ to $20 \mu \mathrm{m})$ as shown in Fig. 7(c). However, it stabilizes in the range of $12 \mu \mathrm{m}-15 \mu \mathrm{m}$ which signifies the need for an appropriate WG length for sensor applications.

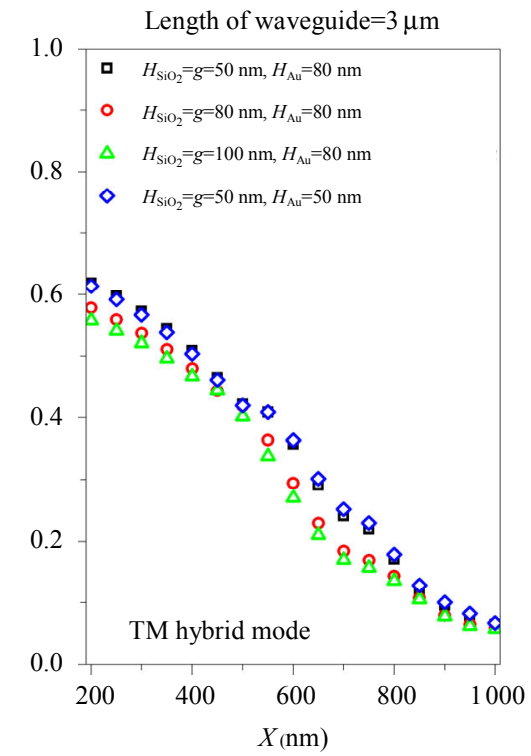

(b)

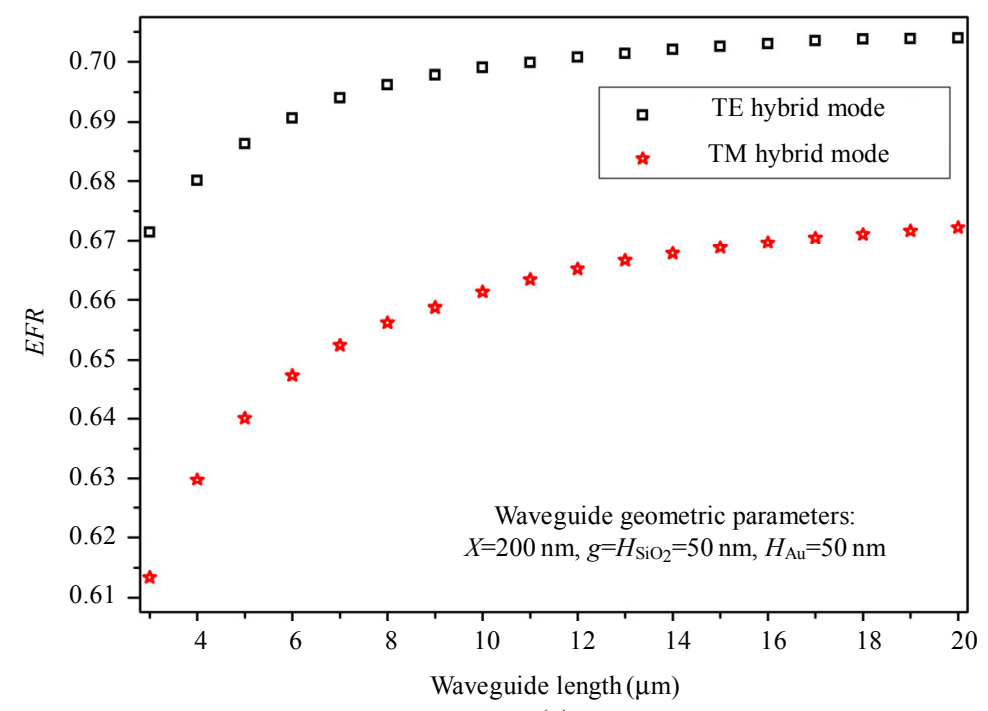

(c)

Fig. 7 EFR versus WG geometry at the fixed WG length: (a) TE hybrid mode, (b) TM hybrid mode, and (c) EFR versus WG length at fixed geometric parameters, i.e., $X=200 \mathrm{~nm}$ and $g=H_{\mathrm{SiO}_{2}}=H_{\mathrm{Au}}=50 \mathrm{~nm}$. 


\section{7. $\mathrm{CH}_{2}$ gas sensing via Lambert-Beer's formulism}

The sensing performance of the polarization-insensitive HPWG optimized at $3.392 \mu \mathrm{m}$ is analyzed for $\mathrm{CH}_{4}$ gas detection. $\mathrm{CH}_{4}$ possesses weak absorption lines at $1.33 \mu \mathrm{m}$ and $1.66 \mu \mathrm{m}$ and demonstrates a strong fundamental absorption at $3.392 \mu \mathrm{m}$. The absorption of $\mathrm{CH}_{4}$ at $3.3 \mu \mathrm{m}$ is approximately 100 times stronger than that in its overtone band at $1.65 \mu \mathrm{m}$. According to Lambert-Beer's law, the light propagating through the gas experiences power decays if it contains the absorption line of that gas. It can be calculated as follows:

$$
P_{o}=P_{i} \exp (\varepsilon \eta C L-\alpha L)
$$

where $P_{i}$ is the input power. In this analysis, $P_{i}$ is fixed at $15 \mathrm{~mW} ; \varepsilon$ is the gas absorption coefficient which is equal to $0.00083 \mathrm{~atm}^{-1} \cdot \mu \mathrm{m}^{-1}$ for $3.392 \mu \mathrm{m}$ line of a He-Ne laser [13]; $\eta$ is EFR; $C$ is the concentration of $\mathrm{CH}_{4}$ gas; $L$ is the length of light through the gas; $\alpha$ is the intrinsic loss of the WG; $P_{o}$ is the power at the output of the WG. The power is articulated in decibel $(\mathrm{dB})$, therefore: Power $(\mathrm{dB})=$ $10 \times \lg \left(\frac{P_{o}}{P_{i}}\right)$. The gas sensing process is revealed in Fig. 8(a). It can be seen that the gas molecules encircle the WG sensor which will then absorb the light of the propagating mode via the evanescent field. As a result, the power at the output of the WG will be diminished. The WG sensor response is calculated at fixed geometric parameters, i.e., $X=$ $200 \mathrm{~nm}, g=H_{\mathrm{SiO} 2}=H_{\mathrm{Au}}=50 \mathrm{~nm}$. For these WG geometric parameters, we can acquire high $E F R$ and $S_{\text {mode }}$ at the cost of the high intrinsic loss of the TE hybrid and TM hybrid modes. As shown in Fig. 7(c), $\eta$ highly depends on the WG length, therefore, we select three WG lengths for instance: $3 \mu \mathrm{m}, 10 \mu \mathrm{m}$, and $20 \mu \mathrm{m}$. From Lambert-Beer's law, it is perceptible that $C$ is directly proportional to the decay in the output power that is why the gas concentration is taken in the range of $0-100 \%$. The transmitted power decreases as $C$ increases because a large number of gas analytes can absorb more light which results in the reduction of the output power. Moreover, the intrinsic loss of the WG reduces the power of the TE hybrid and TM hybrid modes at the input to $0.58 \mathrm{~dB}$ and $1.51 \mathrm{~dB}$, respectively. A WG with a longer length $(20 \mu \mathrm{m})$ shows a strong power decay as shown in Figs. 8(b) and 8(c). For instance, a $3 \mathrm{~dB}$ power decay in the $20-\mu \mathrm{m}$-long WG can be obtained for both the TE and TM hybrid modes for $60 \%$ gas concentration. From this discussion, it is suggested that the length of the WG sensor should be longer than $10 \mu \mathrm{m}$ so as to have a $3 \mathrm{~dB}$ power decay at any given polarization.
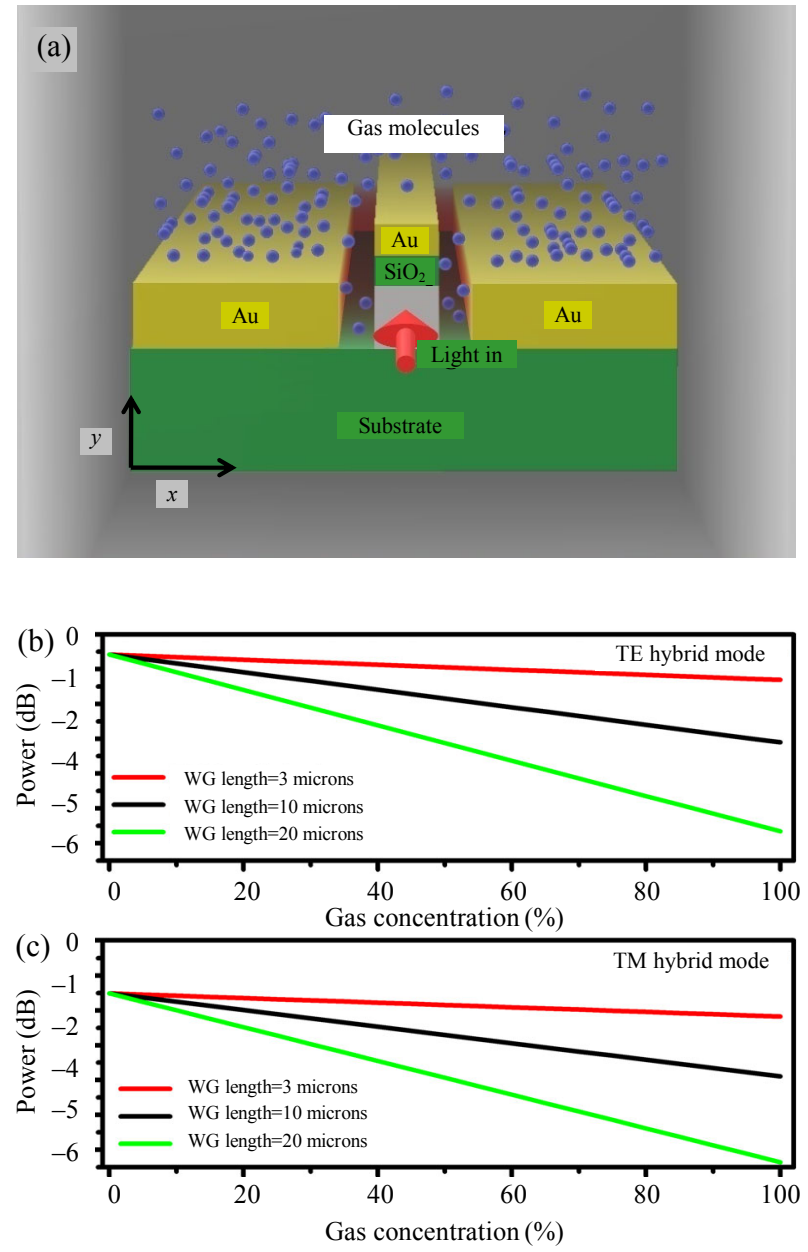

Fig. 8 Schematic representation of (a) gas sensor cell, (b) power variation versus gas concentration for the TE hybrid mode, and (c) power variation versus gas concentration for the TM hybrid mode. 


\section{Suggested fabrication steps of the HPWG}

The polarization-insensitive HPWG is the combination of vertical and horizontal HPWGs, which can be fabricated in two steps. In the first step, a horizontal oriented HPWG is manufactured which supports the TM hybrid mode. This can be obtained as follows: at first, the $50 \mathrm{~nm}$ thin $\mathrm{SiO}_{2}$ layer is deposited on the SOI wafer followed by a $50 \mathrm{~nm}$ of the $\mathrm{Au}$ layer on the top. A suitable photoresist (PR) with a suitable thickness is spun on the sample which will act as a mask. The WG patterns are transferred on the PR which protects the WG core region during the etching process. The WG structures can be formed via the reactive ion etching (RIE) technique. The vertical HPWG is obtained in the following steps: PR is spin-coated on the WG structure and the desired part is patterned to protect the core region. A $200 \mathrm{~nm}$ Au layer is deposited on the sample followed by a lift-off removing the unwanted $\mathrm{Au}$ along with $\mathrm{PR}$ sitting next to the vertical HPWG structure. As a result, a polarization-insensitive HPWG structure is developed. The proposed fabrication steps are presented in Fig. 9 .

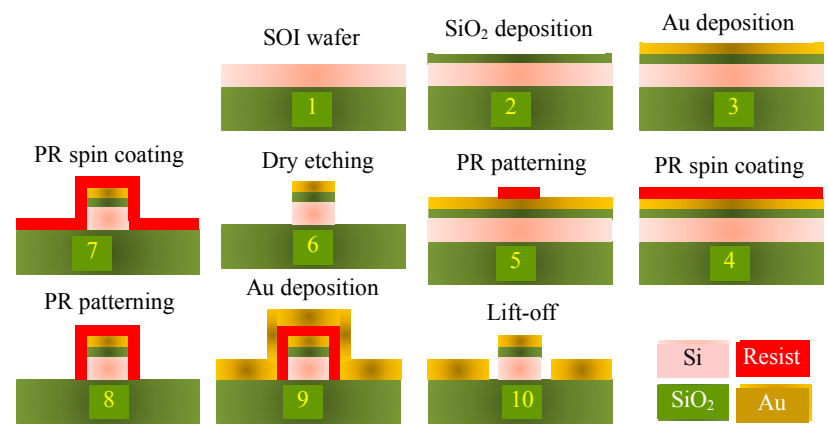

Fig. 9 Proposed fabrication steps of the HPWG.

\section{Conclusions}

We present an attractive design of a polarization-insensitive hybrid plasmonic WG for evanescent field absorption gas sensing applications. The WG geometry is optimized at the $3.392 \mu \mathrm{m}$ wavelength which corresponds to the absorption line of methane gas. The electric field distribution, $n_{\text {eff, }}$ $S_{\text {mode, }}$ and EFR are calculated via the $2 \mathrm{D}$ and $3 \mathrm{D}$ finite element methods. We obtain $S_{\text {mode }}$ and $E F R$ of 0.94 and 0.704 for the TE hybrid mode which are slightly higher than those of the TM hybrid mode i.e., 0.86 and 0.67 , respectively. The TE and TM hybrid mode power dissipation of $3 \mathrm{~dB}$ is obtained for $20-\mu \mathrm{m}$-long hybrid plasmonic WG for the $60 \%$ gas concentration. We believe that the proposed WG scheme can be applied to those situations where strong polarization control is an issue or even can be used in typical applications as a highly sensitive gas absorption sensor.

\section{Acknowledgment}

This work was financially supported by the Russian Foundation for Basic Research (Grant No. 16-29-09528_ofi_m) for numerical calculations, by the Ministry of Science and Higher Education within the State assignment FSRC «Crystallography and Photonics» RAS (Grant No. 007-GZ/Ch3363/26) for theoretical results.

Open Access This article is distributed under the terms of the Creative Commons Attribution 4.0 International License (http://creativecommons.org/licenses/by/4.0/), which permits unrestricted use, distribution, and reproduction in any medium, provided you give appropriate credit to the original author(s) and the source, provide a link to the Creative Commons license, and indicate if changes were made.

\section{References}

[1] Y. F. Chou Chau, C. T. Chou Chao, H. J. Huang, M. R. Rahimi Kooh, N. T. R. N. Kumara, C. M. Lim, et al., "Perfect dual-band absorber based on plasmonic effect with the cross-hair/nanorod combination," Nanomaterials, 2020, 10(3): 493-1-493-15.

[2] M. A. Butt, N. L. Kazanskiy, and S. N. Khonina, "Highly sensitive refractive index sensor based on plasmonic bow tie configuration," Photonic Sensors, 2020, 10(3): 223-232.

[3] Y. F. Chou Chau, C. T. Chou Chao, C. M. Lim, H. J. Huang, and H. P. Chiang, "Deploying tunable metal-shell/dielectric core nanorod arrays as the 
virtually perfect absorber in the near-infrared regime," ACS Omega, 2018, 3(7): 7508-7518.

[4] Y. F. Chou Chau, C. T. Chou Chao, H. P. Chiang, C. M. Lim, N. Y. Voo, and A. H. Mahadi, "Plasmonic effects in composite metal nanostructures for sensing applications," Journal of Nanoparticle Research, 2018, 20(7): 190-1-190-13.

[5] H. H. Qazi, A. B. Mohammad, and M. Akram, "Recent progress in optical chemical sensors," Sensors, 2012, 12(12): 16522-16556.

[6] P. Gruber, M. P. C. Marques, N. Szita, and T. Mayr, "Integration and application of optical chemical sensors in microbioreactors," Lab on a Chip, 2017, 17(16): 2693-2712.

[7] M. Majdinasab, K. Mitsubayashi, and J. L. Marty, "Optical and electrochemical sensors and biosensors for the detection of quinolones," Trends in Biotechnology, 2019, 37(8): 898-915.

[8] J. Hodgkinson and R. P. Tatam, "Optical gas sensing: a review," Measurement Science and Technology, 2013, 24(1): 012004-1-012004-59.

[9] J. Y. Jo, Y. S. Kwon, J. W. Lee, J. S. Park, B. H. Rho, and W. II. Choi, "Acute respiratory distress due to methane inhalation," Tuberculosis and Respiratory Diseases, 2013, 74(3): 120-123.

[10] J. G. Speight, Chapter 2: origin and production in natural gas: a basic handbook. Oxford: Gulf Professional Publishing, 2018: 25-57.

[11] M. A. Butt, S. N. Khonina, and N. L. Kazanskiy, "Silicon on silicon dioxide slot waveguide evanescent field gas absorption sensor," Journal of Modern Optics, 2018, 65(2): 174-178.

[12] M. A. Butt, S. N. Khonina, and N. L. Kazanskiy, "Modelling of Rib channel waveguides based on silicon-on-sapphire at $4.67 \mu \mathrm{m}$ wavelength for evanescent field gas absorption sensor," Optik, 2018, 168: 692-697.

[13] M. A. Butt, S. A. Degtyarev, S. N. Khonina, and N. L. Kazanskiy, "An evanescent field absorption gas sensor at mid-IR $3.39 \mu \mathrm{m}$ wavelength," Journal of Modern Optics, 2017, 64(18): 1892-1897.

[14] A. Novack, M. Streshinsky, R. Ding, Y. Liu, A. E. J. Lim, G. Q. Lo, et al., "Progress in silicon platforms for integrated optics," Nanophotonics, 2014, 3(4-5): 205-214.

[15] M. A. Butt, S. N. Khonina, and N. L. Kazanskiy, "Optical elements based on silicon photonics," Computer Optics, 2019, 43(6): 1079-1083.

[16] B. L. Bachim and T. K. Gaylord, "Polarization-dependent loss and birefringence in long-period fiber gratings," Applied Optics, 2003, 42(34): 6816-6823.

[17] M. Kumar, "Polarization insensitive hollow optical waveguide," Optics Communications, 2012, 285(9): 2360-2362.

[18] T. Baba and Y. Kokubun, "New polarization-insensitive antiresonant reflecting optical waveguide," IEEE Photonics Technology Letters, 1989, 1(8): 232-234.

[19] Q. Han, J. St-Yves, Y. Chen, M. Menard, and W. Shi, "Polarization-insensitive silicon nitride arrayed waveguide grating," Optics Letters, 2019, 44(16): 3976-3979.

[20] N. L. Kazanskiy, S. N. Khonina, and M. A. Butt, "Plasmonic sensors based on Metal-insulator-metal waveguides for refractive index sensing applications: A brief review," Physica E: Low-Dimensional Systems and Nanostructures, 2020, 117: 113798-1-113798-10.

[21] M. A. Butt, S. N. Khonina, and N. L. Kazanskiy, “A multichannel metallic dual nano-wall square split-ring resonator: design analysis and applications," Laser Physics Letters, 2019, 16(12): 126201-1-126201-7.

[22] N. T. R. N. Kumara, Y. F. Chou Chau, J. W. Huang, H. J. Huang, C. T. Lin, and H. P. Chiang, "Plasmonic spectrum on $1 \mathrm{D}$ and $2 \mathrm{D}$ periodic arrays of rod-shape metal nanoparticle pairs with different core patterns for biosensor and solar cell applications," Journal of Optics, 2016, 18(11): 115003-1-115003-7.

[23] Y. F. Chou Chau, K. H. Chen, H. P. Chiang, C. M. Lim, H. J. Huang, C. H. Lai, et al., "Fabrication and characterization of a metallic-dielectric nanorod array by nanosphere lithography for plasmonic sensing application," Nanomaterials, 2019, 9(12): 1691-1-1691-15.

[24] Y. Fang and M. Sun, "Nanoplasmonic waveguides: towards applications in integrated nanophotonic circuits," Light: Science and Applications, 2015, 4(6): e294-1-e294-11.

[25] M. A. Butt, S. N. Khonina, and N. L. Kazanskiy, "Ultra-short lossless plasmonic power splitter design based on metal-insulator-metal waveguide," Laser Physics, 2019, 30(1): 016201-1016201-6.

[26] Y. F. Chou Chau, C. T. Chou Chao, H. J. Huang, U. Anwar, C. M. Lim, N. Y. Voo, et al., "Plasmonic perfect absorber based on metal nanorod arrays connected with veins," Results in Physics, 2019, 15: 102567-1-102567-6.

[27] M. A. Butt, S. N. Khonina, and N. L. Kazanskiy, “A plasmonic colour filter and refractive index sensor applications based on metal-insulator-metal square $\mu$-ring cavities," Laser Physics, 2020, 30(1): 016205-1-016205-5.

[28] D. Chandler-Horowitz, "High-accuracy, midinfrared $\left(450 \mathrm{~cm}^{-1} \leq \omega \leq 4000 \mathrm{~cm}^{-1}\right)$ refractive index values of silicon," Journal of Applied Physics, 2005, 97(12): 123526-1-123526-8.

[29] I. H. Malitson, "Interspecimen comparison of the refractive index of fused silica," Journal of the Optical Society of America, 1965, 55(10): 1205-1209. 
[30] R. L. Olmon, B. Slovick, T. W. Johnson, D. Shelton, S. H. Oh, G. D. Boreman, et al., "Optical dielectric function of gold," Physical Review B, 2012, 86(23): 235147-1-235147-9.

[31] M. Odeh, K. Twayana, K. Sloyan, J. E. Villegas, S. Chandran, and M. S. Dahlem, "Mode sensitivity analysis of subwavelength grating slot waveguides," IEEE Photonics Journal, 2019, 11(5): 2700210-1-2700210-10.

[32] D. F. Swinehart, "The beer-lambert law," Journal of Chemical Education, 1962, 39(7): 333-335.

[33] S. M. Babin and R. M. Sova, "Preliminary development of a fiber optic sensor for measuring bilirubin," Analytical Chemistry Insights, 2014, 9: 59-65.
[34] S. N. Khonina, N. L. Kazanskiy, and M. A. Butt, "Evanescent field ratio enhancement of a modified ridge waveguide structure for methane gas sensing application," IEEE Sensors Journal, 2020, 20(15): 8469-8476.

[35] Y. Qiao, J. Tao, C. H. Chen, J. Qiu, Y. Tian, X. Hong, et al., "A minature on-chip methane sensor based on an ultra-low loss waveguide and a micro-ring resonator filter," Micromachines, 2017, 8(15): 160-1-160-9.

[36] C. Ranacher, C. Consani, N. Vollert, A. Tortschanoff, M. Bergmeister, T. Grille, et al., "Characterization of evanescent field gas sensor structures based on silicon photonics," IEEE Photonics Journal, 2018, 10(5): 2700614-1-2700614-14. 